\title{
Decreased expression levels of Nurr1 are associated with chronic inflammation in patients with type 2 diabetes
}

\author{
YING XU $^{1 *}$, QI HUANG ${ }^{1 *}$, WENFANG ZHANG ${ }^{2}$, YAPING WANG ${ }^{2}$, QINGLING ZENG ${ }^{2}$, \\ CHUNYAN HE $^{2}$, JUNLI XUE ${ }^{1}$, JIN CHEN $^{1}$, XUEMEI HU ${ }^{1}$ and YANCHENG XU ${ }^{1}$ \\ ${ }^{1}$ Department of Endocrinology, Zhongnan Hospital of Wuhan University, Wuhan, Hubei 430071; \\ ${ }^{2}$ Department of Endocrinology, The Fifth Hospital of Wuhan, Wuhan, Hubei 430071, P.R. China
}

Received August 9, 2014; Accepted April 20, 2015

DOI: $10.3892 / \mathrm{mmr} .2015 .4105$

\begin{abstract}
Chronic inflammation is associated with insulin resistance, a characteristic of type 2 diabetes (T2D). Nuclear receptor-related protein 1 (Nurrl) can regulate inflammation, dependent on the nature of individual diseases. However, whether Nurrl regulates chronic inflammation during the pathogenic process of T2D in humans remains to be fully elucidated. The present study aimed to investigate the potential association between the expression of Nurrl in peripheral blood mononuclear cells (PBMCs) and inflammation in patients with T2D. The levels of plasma tumor necrosis factor (TNF) $\alpha$ and interleukin (IL)-6, the relative expression levels of Nurrl, and glycogen synthase kinase (GSK)-3 $\beta$ phosphorylation in PBMCs from 40 patients with T2D and 40 healthy controls (HC group) were examined, and their potential association with clinical measures were analyzed. The expression levels of Nurrl, induced by high glucose and palmitic acid, were assessed in the PBMCs from the HC group. Compared with the $\mathrm{HC}$ group, significantly higher levels of plasma TNFa and IL- 6 were correlated positively with the degree of insulin resistance in the T2D patients. However, significantly lower expression levels of Nurrl and GSK-3 $\beta$ phosphorylation in the PBMCs were correlated negatively with the levels of TNF $\alpha$, IL-6, fasting insulin and insulin resistance in the T2D patients. Treatment of the PBMCs with high glucose or palmitic acid inhibited the expression of Nurrl in a dose- and time-dependent manner. Therefore, decreased expression levels of Nurrl were associated with chronic inflammation and insulin resistance in patients with T2D.
\end{abstract}

Correspondence to: Dr Yancheng Xu, Department of Endocrinology, Zhongnan Hospital of Wuhan University, 169 Donghu Road, Wuhan, Hubei 430071, P.R. China

E-mail: oxyc@163.com

*Contributed equally

Key words: Nurr1, chronic inflammation, insulin resistance, type 2 diabetes

\section{Introduction}

Type 2 diabetes (T2D) is a metabolic disorder, which affects $>300,000,000$ individuals worldwide, and its incidence is increasing, particularly in developing countries, including China (1). At present, there are several therapeutic strategies available for the intervention of patients with T2D. However, the long term efficacy of anti-diabetic therapies requires improvement, and potential hyperglycemia-associated complications remain a leading cause of diabetes-associated mortality (2). The pathogenesis of T2D remains to be fully elucidated, therefore, understanding the pathogenic process of T2D may promote the development of effective therapies for patients with T2D.

Previous studies have suggested that chronic inflammation is associated with the development of T2D $(3,4)$. Activated macrophages, $T$ cells and $B$ cells infiltrate into the adipose and muscle tissues, contributing to the development of insulin resistance, a hallmark of the pathogenesis of T2D (5-7). Furthermore, activated inflammatory cells secrete pro-inflammatory cytokines, including tumor necrosis factor (TNF) $\alpha$, interleukin (IL)-6, which positively regulate insulin resistance. Higher levels of serum pro-inflammatory cytokines are detected in T2D patients (8). However, the precise regulation of chronic inflammation during the pathogenesis of T2D in humans remains to be fully elucidated.

Nuclear receptor-related protein 1 (Nurrl) is one member of the nuclear receptor subfamily and regulates several physiological functions (9). In addition to the central nervous system, Nurr1 is expressed in adipose tissues, the liver, skeletal muscles, and heart tissues, and is closely associated with the development of metabolic diseases in humans (10). Previous studies have demonstrated that Nurr1 inhibits muscular cell proliferation and inflammation (11-13), and protects against activated microglial cell-mediated neuronal cytotoxicity $(14,15)$. Furthermore, Nurrl also inhibits the production of pro-inflammatory cytokines by macrophages and microglial cells $(16,17)$. Nurrl can interact with the nuclear factor (NF)- $\mathrm{kB}$ pathway to downregulate inflammation, which is dependent on the activity of glycogen synthase kinase (GSK)-3 $\beta$ (18-20). However, high expression levels of Nurr1 are detected in inflamed human synovial tissue, psoriatic skin, atherosclerotic lesions, lung and colorectal cancer cells (21-24). Therefore, 
Nurr1 may have a dual function in regulating inflammatory process, depending on the nature of the individual diseases. Whether Nurr1 regulates chronic inflammation during the pathogenic process of T2D in humans remains to be elucidated, and the association between the expression levels of Nurr1 and the production of inflammatory cytokines in human peripheral blood mononuclear cells (PBMCs) from diabetic patients has not been investigated.

In the present study, the relative expression levels of Nurrl in human PBMCs and the level of serum pro-inflammatory cytokines were examined in 40 newly diagnosed T2D patients and 40 healthy controls, and the potential association between the expression levels of Nurr1, serum cytokine levels and clinical measures were investigated.

\section{Materials and methods}

Subjects. A total of 40 patients, newly diagnosed with T2D and 40 age and gender-matched healthy control individuals (HC) were recruited at Zhongnan Hospital of Wuhan University (Wuhan, China) between October 2012 and February 2013. The patients with T2D were diagnosed, according to the diagnostic criteria of the American Diabetes Association 2003 (25). Individuals were excluded if they had a history of hypoglycemia, ketoacidosis, hypertension, cardiovascular disease, liver disease, any autoimmune disease or a recent infectious disease. Written informed consent was obtained from each individual involved, and the experimental procedure was approved by the Ethics Committee of Zhongnan Hospital of Wuhan University (Approval no. 2012012).

Clinical measurements and laboratory assessments. The body weight, height and body mass index of each individual subject were measured. Individual subjects were fasted for at least $12 \mathrm{~h}$, and median cubital venous blood samples (4 $\mathrm{ml}$ per patient) were obtained. The concentrations of fasting plasma glucose (FPG), total triglycemia (TG), total cholesterol (TC), high density lipoprotein (HDL), low density lipoprotein, (LDL) as well as the levels of plasma alanine aminotransferase (ALT) were measured by scatter turbidimetry using a Siemens Special Protein Analysis instrument (Siemens Healthcare Diagnostics Products, GmbH, Marburg, Germany). The plasma concentrations of fasting insulin (FINS) were measured by chemiluminescent immunoassay, using the Insulin (Human) CLIA kit, according to manufacturer's instructions (Abnova, Walnut, CA, USA). The values of homeostasis model assessment of insulin resistance (HOMA-IR) in individual subjects were calculated, according to the formula: Fasting insulin (microU/L) x fasting glucose (nmol/l)/22.5 (26).

Isolation of human PBMCs and stimulation. Fasting blood samples were obtained from individual subjects and their PBMCs were prepared by density gradient centrifugation using Ficoll-Hypaque (PAA Laboratories GmbH, Pasching, Austria). PBMCs were used to analyze the levels of the Nurr1 mRNA transcript using reverse transcription-quantitative polymerase chain reaction (RT-qPCR), and Nurrl protein expression and GSK-3 $\beta$ phosphorylation using western blot analysis.

In addition, a quantity of the PBMCs $\left(3 \times 10^{5} / \mathrm{ml}\right)$ were stimulated with, or without, 250 or $500 \mu \mathrm{M}$ palmitic acid, or with
16.7 or $33.3 \mathrm{mM}$ glucose (Sigma-Aldrich, St Louis, MO, USA) in $10 \%$ fetal bovine serum (FBS) RPMI-1640 (Invitrogen Life Technologies, Carlsbad, CA, USA) at $37^{\circ} \mathrm{C}$ for $45 \mathrm{~min}$ and $24 \mathrm{~h}$, respectively. The supernatants were then harvested for analysis of cytokines, and the cells were collected for the extraction of RNA and proteins for further analyses.

$R T$ - $q P C R$. The levels of the Nurr1 mRNA transcripts relative to GAPDH in individual PBMC samples were determined using RT-qPCR. Briefly, total RNA was isolated from the PBMCs using TRIzol reagent (Invitrogen Life Technologies), and reverse-transcribed into cDNA using a RevertAid ${ }^{\mathrm{TM}}$ First Strand cDNA Synthesis kit (Fermentas, Pittsburgh, PA, USA), according to the manufacturer's instructions. The qPCR was performed using SYBR Green PCR Master Mix and specific primers on a 7500 Fast Real-Time PCR system (Applied Biosystems, Foster City, CA, USA), and 40 ng cDNA per sample was used for the template. The following primer sequences were used: Nurr1 forward 5'-CCTTGTGTTCAGGCGCAGTAT-3' and reverse 5'-GTGGCAGTGATTTCAGTGTTGGT-3' (158bp); TNF- $\alpha$ forward 5'-GCCAGCTCCCTCTATTTATG-3' and reverse 5'-TGGTCACCAAATCAGCATTG-3' for TNF- $\alpha$ (272 bp), and GAPDH forward 5'-GGC TGAGAACCGGAAGCTTGTCAT-3' and reverse 5'-CAGCCT TCTCCATGCTGGTGGTGAAGA-3' for (314 bp). The amplification was performed at $95^{\circ} \mathrm{C}$ for $5 \mathrm{~min}$ followed by 40 cycles of $94^{\circ} \mathrm{C}$ for $15 \mathrm{sec}, 55^{\circ} \mathrm{C}$ for $20 \mathrm{sec}, 72^{\circ} \mathrm{C}$ for $20 \mathrm{sec}$, followed by $72^{\circ} \mathrm{C}$ for $7 \mathrm{~min}$. The levels of mRNA transcripts were analyzed using the $2^{-\Delta \Delta \mathrm{Ct}}$ method (27).

Western blot analysis. The collected PBMC samples were lyzed in radioimmunoprecipitation assay buffer containing $1 \%$ Triton $\mathrm{X}-100,50 \mathrm{mM} \mathrm{KCl}, 25 \mathrm{mM}$ Hepes ( $\mathrm{pH} 7.8$ ), $10 \mu \mathrm{g} / \mathrm{ml}$ leupeptin, $20 \mu \mathrm{g} / \mathrm{ml}$ aprotinin, $125 \mu \mathrm{M}$ dithiothreitol, $1 \mathrm{mM}$ phenylmethanesulfonyl fluoride and $1 \mathrm{mM}$ sodium orthovanadate (Sigma-Aldrich), and centrifuged at $12,000 \mathrm{x}$ g for $15 \mathrm{~min}$ at $4^{\circ} \mathrm{C}$. Following quantification of the protein concentrations using a bicinchoninic acid assay (Santa Cruz Biotechnology, Inc., Dallas, TX, USA), the individual cell lysates (40 $\mu \mathrm{g} /$ lane) were separated by $12 \%$ sodium dodecyl sulfate polyacrylamide gel electrophoresis and transferred onto polyvinylidene fluoride membranes (EMD Millipore, Billerica, MA, USA). The membranes were blocked with $5 \%$ fat-free dry-milk in Tris-buffered saline with $0.1 \%$ Tween 20 and incubated with the following antibodies: Mouse monoclonal anti-Nurr1 (cat. no. ab54366; 1:1,000; Abcam, Cambridge, MA, USA), rabbit polyclonal anti-pho-GSK-3 $\beta$ (cat. no. ab75745; 1:1,000; Abcam), goat polyclonal anti-GAPDH (cat. no. sc-20356; 1:10,00; Santa Cruz Biotechnology, Inc.) and rabbit polyclonal anti- $\beta$-actin (cat. no. sc1616R; 1:1,000; Santa Cruz Biotechnology, Inc.) overnight at $4{ }^{\circ} \mathrm{C}$, respectively. Following washing twice, the bound antibodies were detected using horseradish peroxidase-conjugated anti-rabbit (cat. no. sc-2030; 1:3,000; Santa Cruz Biotechnology, Inc.), or anti-mouse (cat. no. sc-2005; 1:3,000; Santa Cruz Biotechnology, Inc.)at room temperature for $1 \mathrm{~h}$, and visualized using enhanced chemiluminescence (Santa Cruz Biotechnology, Inc.). Purified mouse, or rabbit immunoglobulin $\mathrm{G}$ were used as negative controls. The levels of targeting proteins relative to $\beta$-actin or GAPDH were 
Table I. Demographic and clinical characteristics of the recruited patients and healthy control subjects.

\begin{tabular}{lccc}
\hline Variable & Diabetic group & Control group & P-value \\
\hline Age (years) & $53.03 \pm 8.3$ & $51.05 \pm 11.45$ & $\mathrm{NS}$ \\
Male/female & $24 / 16$ & $18 / 22$ & $\mathrm{NS}$ \\
Body mass index & $22.80 \pm 2.79$ & $21,87 \pm 2.18$ & $\mathrm{NS}$ \\
Total cholesterol $(\mathrm{mmol} / \mathrm{l})$ & $5.56 \pm 2.45$ & $4.80 \pm 0.94$ & $\mathrm{NS}$ \\
Total triglycemia $(\mathrm{mmol} / \mathrm{l})$ & $2.07 \pm 0.75$ & $1.15 \pm 0.59$ & $<0.01$ \\
High density lipoprotein $(\mathrm{mmol} / \mathrm{l})$ & $1.02 \pm 0.25$ & $1.34 \pm 0.31$ & $<0.01$ \\
Low density lipoprotein $(\mathrm{mmol} / \mathrm{l})$ & $3,02 \pm 0.66$ & $2.84 \pm 0.51$ & $\mathrm{NS}$ \\
Fasting insulin $(\mu \mathrm{U} / \mathrm{ml})$ & $16.26 \pm 3.95$ & $6.60 \pm 2.38$ & $<0.01$ \\
Fasting plasma glucose $(\mathrm{mmol} / \mathrm{l})$ & $9.01 \pm 1.62$ & $4.96 \pm 0.43$ & $1.46 \pm 0.57$ \\
HOMA-IR & $6.56 \pm 1.98$ & $21.23 \pm 12.33$ & $<0.01$ \\
Alanine aminotransferase $(\mathrm{U} / \mathrm{l})$ & $22.23 \pm 10.18$ & $49.63 \pm 13.92$ & $\mathrm{NS}$ \\
Tumor necrosis factor $\alpha(\mathrm{pg} / \mathrm{ml})$ & $75.29 \pm 16.52$ & $10.68 \pm 5.83$ & $<0.01$ \\
Interleukin-6 $(\mathrm{pg} / \mathrm{ml})$ & $19.32 \pm 7.12$ & & $<0.01$ \\
\hline
\end{tabular}

NS, no statistical significance.

determined using Alpha EaseFC 4.0 (Alpha Innotech, San Leandro, CA, USA).

ELISA. The levels of IL-6 and TNF $\alpha$ in individual plasma samples were determined using specific ELISA kits (Boster Statems, Inc., Wuhan, China), according to the manufacturer's instructions. The limitations of detection for plasma IL-6 and $\mathrm{TNF} \alpha$ were $1.8 \mathrm{pg} / \mathrm{ml}$, and $13.3 \mathrm{pg} / \mathrm{ml}$, respectively.

Statistical analysis. Data are presented as the median (range) or the mean \pm standard deviation. Differences between groups were analyzed using Student's t-test for normally distributed data or using a Wilcoxon signed-rank test for skewed data. The differences in the category data between these two groups were analyzed using a $\chi^{2}$-square test. The potential association between variants was analyzed using Spearman's rank correlation coefficient. All statistical analyses were performed using the SPSS statistics 16.0 software package. $\mathrm{P}<0.05$ was considered to indicate a statistically significant difference.

\section{Results}

Increased plasma levels of pro-inflammatory cytokines are observed in patients with T2D. To understand the potential regulation of the expression Nurrl on chronic inflammation during the development of T2D, 40 patients with newly diagnosed T2D and 40 age- and gender-matched $\mathrm{HC}$ were recruited to the present study. As shown in Table I, no significant differences were apparent in the distribution of age and gender or BMI between the T2D patients and HC group. The levels of FPG and FINS, and the HOMA IR values in the T2D patients were significantly higher, as compared with that in the $\mathrm{HC}$ group $(\mathrm{P}<0.01$ for all), demonstrating hyperglycemia and compensated increased secretion of insulin and the development of insulin resistance in the T2D patients. Furthermore, although no significant difference was observed in the levels of plasma levels of TC and
LDL-c between the patients and HC group, significantly higher levels of plasma TG and lower levels of HDL-c were detected in the T2D patients $(\mathrm{P}<0.01$ for all), indicating dysfunctional lipid metabolism in T2D patients.

Further analysis revealed that the plasma levels of IL-6 and $\mathrm{TNF} \alpha$ in the T2D patients were significantly higher than those in the $\mathrm{HC}$ group $(\mathrm{P}<0.01$; Fig. 1A and B). Notably, the levels of IL- 6 and TNF $\alpha$ were positively correlated with the values of HOMA-IR in the T2D patients $(r=0.427: \mathrm{P}=0.008$ and $\mathrm{r}=0.39$; $\mathrm{P}=0.015$, respectively), as shown in Fig. $1 \mathrm{C}$ and $\mathrm{D}$. In addition, PBMCs were isolated from the HC and T2D patients, and were stimulated with PMA/ionomycin in vitro, followed by assessment of the relative expression levels of IL-6 and TNF $\alpha$ using western blot analysis. The results revealed that the relative expression levels of IL-6 and TNF $\alpha$ in the PBMCs from the T2D patients were significantly higher than those in the PBMCs from the $\mathrm{HC}$ group (1.08 \pm 0.11 , vs. $0.49 \pm 0.14$ for IL-6; $\mathrm{P}<0.01$ and $1.14 \pm 0.11$, vs. $0.56 \pm 0.09$ for $\mathrm{TNF} \alpha ; \mathrm{P}<0.01)$. These data suggested that high levels of pro-inflammatory cytokines were present in patients with T2D and may be associated with the development of insulin resistance during the development of T2D.

Decreased expression of Nurrl and phosphorylation of GSK-3 $\beta$ are associated with the development of T2D. Nurr1 can downregulate inflammation and pro-inflammatory cytokine production, which depends on activity of GSK-3 $\beta$ in astrocytes and microglial cells $(11,19,21)$. To understand the molecular mechanisms underlying the role of Nurrl in the development of chronic inflammation, the present study analyzed the relative expression levels of Nurrl in the PBMCs from the patients with T2D and the HC group using RT-qPCR and western blot analysis. As shown in Fig. 2A, the relative levels of Nurrl mRNA transcripts in the T2D patients were significantly lower, compared with those in the HC group $(\mathrm{P}=0.012)$. The western blot analysis indicated that the relative protein levels of Nurrl in the PBMCs from the T2D patients were also significantly lower, compared with those in the $\mathrm{HC}(\mathrm{P}=0.0188$; Fig. $2 \mathrm{~B})$. GSK-3 $\beta$ is crucial for 
A

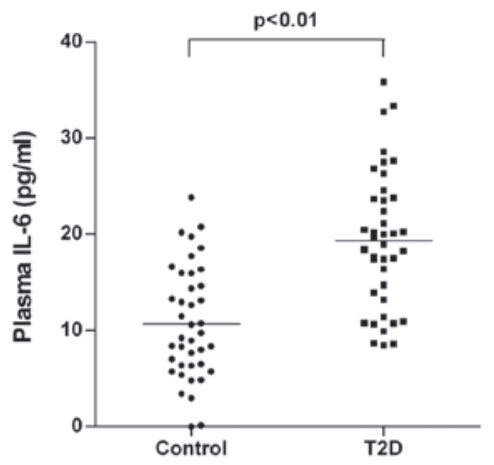

C

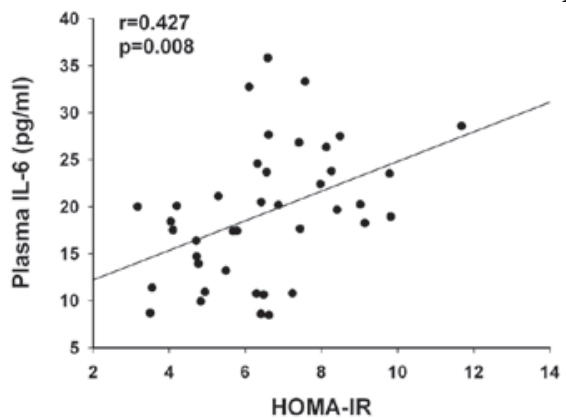

B

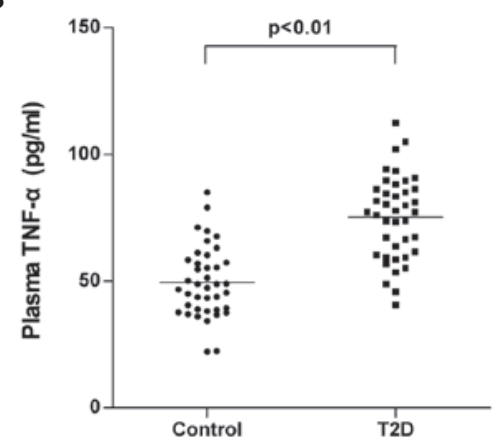

D

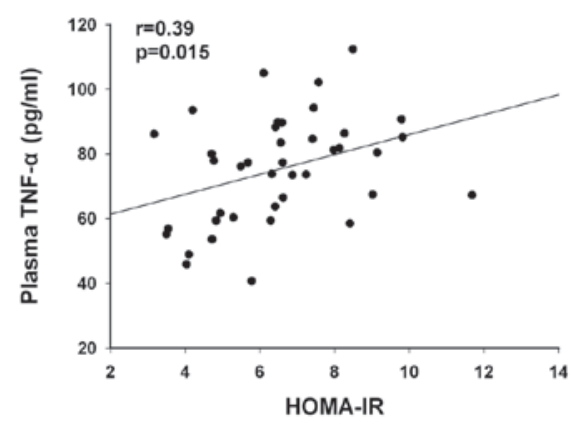

Figure 1. Significantly higher levels of pro-inflammatory cytokines are observed in patients with T2D. The levels of plasma IL-6 and TNF- $\alpha$ in individual subjects were assessed using ELISA, and the potential association between the levels of plasma IL- 6 and TNF- $\alpha$ and the values of HOMA-IR were analyzed using Spearman's rank correlation coefficient. (A) Levels of plasma IL-6. (B) Levels of plasma TNF- $\alpha$. (C) Correlation between the levels of IL-6 and the values of HOMA-IR in T2D patients. (D) Correlation between the levels of TNF- $\alpha$ and the values of HOMA-IR in T2D patients. Data are expressed as the mean values of 40 T2D patients and 40 healthy controls. The horizontal lines indicate the median values. T2D, type 2 diabetes; IL, interleukin; TNF, tumor necrosis factor; HOMA-IR, homeostasis model assessment of insulin resistance.

the recruitment of Nurrl to the promoter of pro-inflammatory cytokines in immunocompetent cells, and the phosphorylation of GSK-3 $\beta$ at tyrosine-216 can enhance its activity (19). To understand the potential role of GSK-3 $\beta$ phosphorylation in regulating the Nurrl-mediated inhibition of cytokine production in human immunocompetent cells, the present study analyzed the relative levels of GSK-3 $\beta$ tyrosine-216 phosphorylation in the PBMCs from the T2D patients and $\mathrm{HC}$ group. The relative levels of GSK-3 $\beta$ tyrosine-216 phosphorylation in the PBMCs from the T2D patients were significantly lower than those in the $\mathrm{HC}$ (Fig. 2C), which may have contributed to the higher levels of pro-inflammatory cytokines in the T2D patients. Therefore, the decreased expression of Nurr1 and phosphorylation of GSK-3 $\beta$ tyrosine-216 may be associated with the development of chronic inflammation and T2D.

Decreased expression levels of Nurrl are inversely correlated with the plasma levels of pro-inflammatory cytokines and the degree of insulin resistance in patients with T2D. To understand the clinical significance of the results, the present study analyzed the association between the relative levels of Nurrl mRNA transcripts in the PBMCs, the levels of plasma cytokines and insulin, and the degree of insulin resistance in the T2D patients. As shown in Fig. 3, the relative levels of Nurrl mRNA transcripts in the PBMCs were positively correlated with the plasma levels of HDL-c $(\mathrm{r}=0.45, \mathrm{P}=0.005)$, but negatively correlated with the plasma levels of IL-6 $(\mathrm{r}=-0.353 ; \mathrm{P}=0.030)$, TNF- $\alpha(-0.426$; $\mathrm{P}=0.008)$, FINS ( $\mathrm{r}=-0.485 ; \mathrm{P}=0.002)$ and HOMA-IR $(\mathrm{r}=-0.424$; $\mathrm{P}=0.008)$. Therefore, decreased expression levels of Nurrl were inversely correlated with the levels of plasma pro-inflammatory cytokines and the degree of insulin resistance in the T2D patients.

High levels of glucose or palmitic acid inhibit the expression of Nurrl in PBMCs. Previous studies have demonstrated that high levels of glucose and saturated fatty acids can enhance the activity of NF-kB and promote the production of TNF- $\alpha$ in human PBMCs (28-30). To further understand the role of hyperglycemia and hyperlipidemia in the development of chronic inflammation, the present study examined the impact of different concentrations of glucose and palmitic acid on the expression levels of Nurrl in the PBMCs from the HC group. As shown in Fig. 4, treatment with $16.7 \mathrm{mmol} / 1$ glucose significantly reduced the expression levels of Nurrl in the PBMCs, compared with the untreated PBMCs. Treatment with a higher concentration of glucose further reduced the expression of Nurrl in the PBMCs. In addition, treatment with a lower dose of glucose for 45 min reduced the expression levels of Nurrl by almost $30 \%$, and treatment with the same dose of glucose decreased the expression levels of Nurrl by $57 \%$ for 24 h. Notably, a similar pattern of reduced levels of Nurrl were observed in the PBMCs following treatment with different concentrations of palmitic acid for varying time-periods in vitro. Therefore, high levels of glucose or palmitic acid inhibited the expression of Nurrl in the PBMCs in a dose- and time-dependent manner.

\section{Discussion}

Chronic inflammation is associated with the development of $\mathrm{T} 2 \mathrm{D}$, and previous studies have demonstrated that abnormal 
A
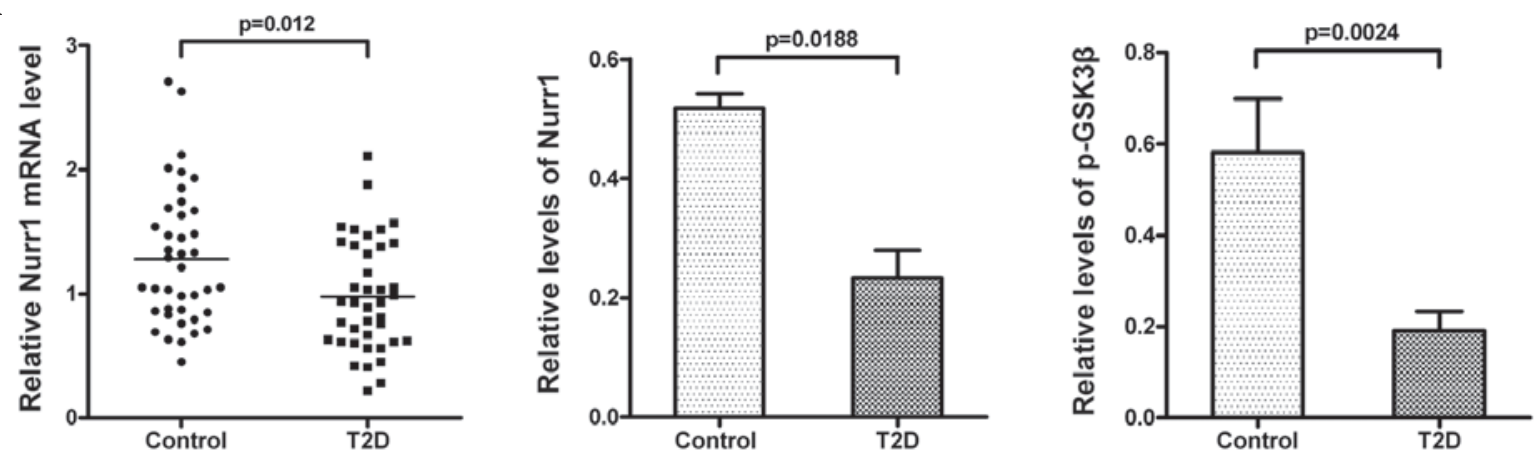

B

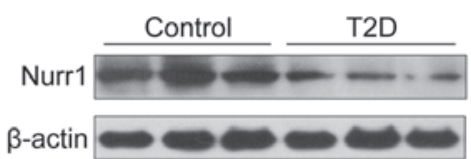

C

p-GSK-3ß

Control

$\mathrm{T} 2 \mathrm{D}$

GAPDH

Figure 2. Decreased expression levels of Nurr1 and GSK-3 $\beta$ phosphorylation in PBMCs from T2D patients. PBMCs were isolated from 40 patients with T2D and 40 healthy controls, and the relative expression levels of Nurrl and GSK-3 $\beta$ phosphorylation were determined using reverse transcription-quantitative polymerase chain reaction and western blot analysis. (A) Relative expression levels of the Nurrl mRNA transcripts. Data are expressed as the mean \pm standard deviation. (B) Relative expression levels of Nurr1 in the PBMCs. (C) Relative levels of GSK-3 $\beta$ phosphorylation in the PBMCs. Nurr1, nuclear receptor-related protein 1; PBMC, peripheral blood mononuclear cell; GSK, glycogen synthase kinase; T2D, type 2 diabetes.

A

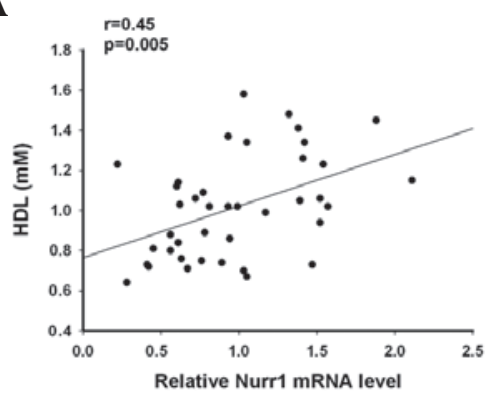

B

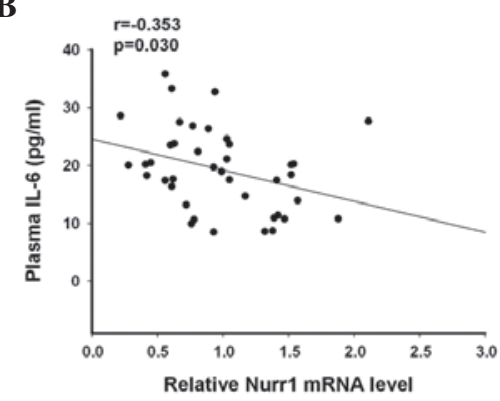

C

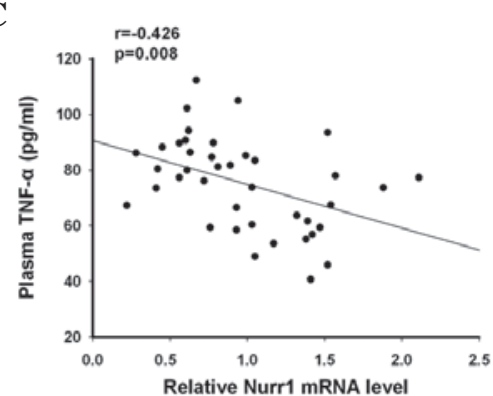

D

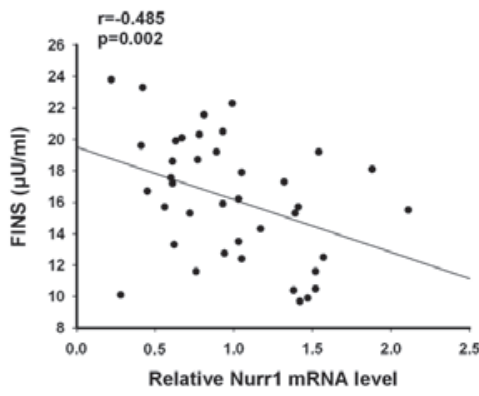

$\mathbf{E}$

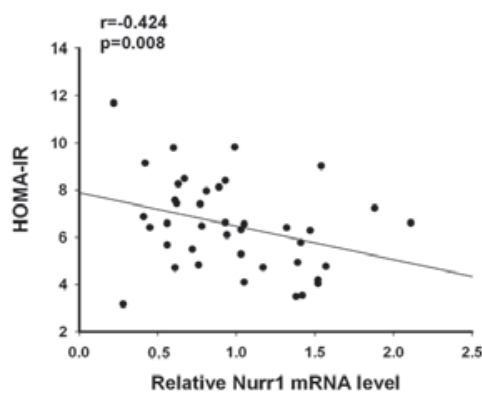

Figure 3. Anaylses of the correlation and potential association between the relative levels of Nurrl mRNA transcripts in the PBMCs with clinical measurements in the T2D patients. Analysis of the potential correlation between the relative levels of Nurrl mRNA transcripts in the PBMCs and the plasma levels of (A) HDL, (B) IL-6, (C) TNF- $\alpha$, (D) FINS and (E) HOMA-IR in 40 patients with T2D were performed using Spearman's rank correlation coefficient. Data are presented as the mean levels of Nurr1 mRNA transcripts against the values of the indicated clinical parameters in individual patients. Nurrl, nuclear receptor-related protein 1; PBMC, peripheral blood mononuclear cell; T2D, type 2 diabetes. HDL, high density lipoprotein; IL, interleukin; TNF, tumor necrosis factor; FINS, fasting insulin; HOMA-IR, homeostasis model assessment of insulin resistance.

exprssion levels of Nurrl are associated with the pathological process of metabolic syndrome in humans $(3,9,10,31)$. However, whether alteration in the expression of Nurr1 in PBMCs occurs in T2D patients, and how these changes in Nurrl are associated with the insulin resistance, a pathogenic basis of T2D remain to be fully elucidated. In the present study, the plasma levels of IL- 6 and TNF- $\alpha$ in the T2D patients were significantly higher than those in the HC group. Furthermore, the significantly higher levels of plasma IL- 6 and TNF- $\alpha$ were positively correlated with the degree of insulin resistance in the T2D patients. These data are consistent with previous observations $(8,32)$ and support the hypothesis that pro-inflammatory cytokines contribute to the development of insulin resistance and T2D in humans.

Nurr1 is considered an inhibitor of inflammation, and its abnormal expression is associated with the development of insulin resistance and metabolic syndrome $(10,31)$. Furthermore, Nurrl can interact with the NF- $\mathrm{B}$ pathway to 
A
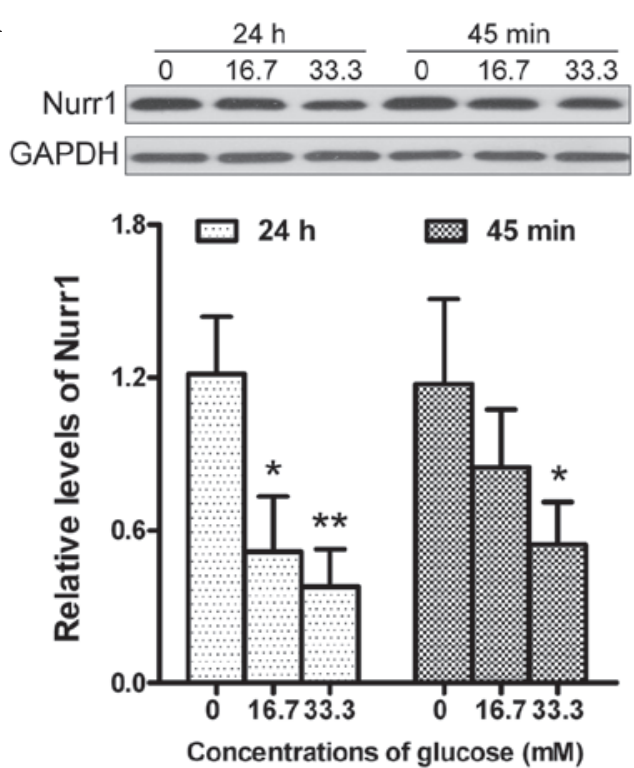

B
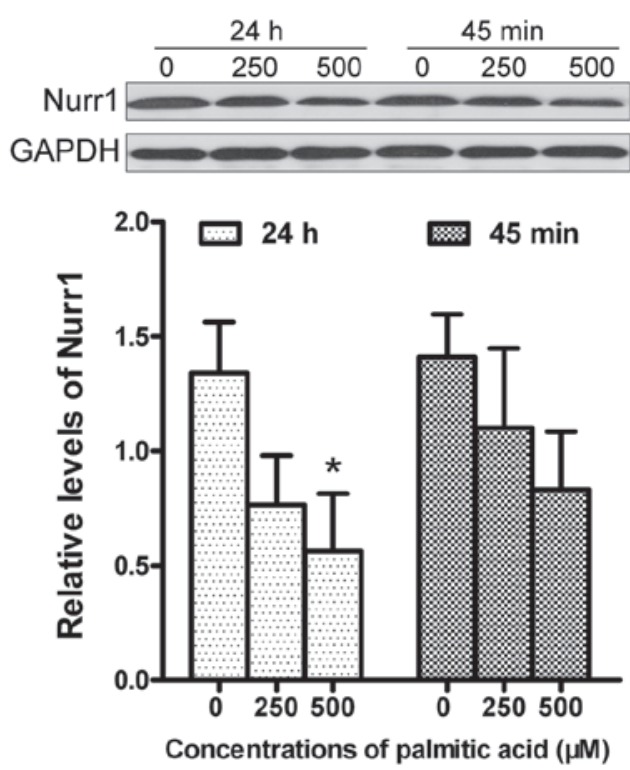

Figure 4. High levels of glucose or palmitic acid inhibit the expression of Nurr1 in PBMCs. The PBMCs were isolated from the healthy control subjects and treated with, or without, the indicated concentrations of (A) glucose or (B) palmitic acid for 45 min or $24 \mathrm{~h}$. The cells were harvested and the relative expression levels of Nurrl in the PBMCs were determined using western blot analysis. Data are expressed as the mean \pm standard deviation from three separate experiments. ${ }^{*} \mathrm{P}<0.05 ;{ }^{* *} \mathrm{P}<0.01$, vs. untreated cells. Nurr1, nuclear receptor-related protein 1 ; PBMC, peripheral blood mononuclear cell; T2D, type 2 diabetes.

downregulate inflammation, which is dependent on GSK-3 $\beta$ phosphorylation (19). In the present study, the expression levels of Nurr1 in the PBMCs from the T2D patients were significantly lower than those in the HC group. Similarly, the relative levels of GSK- $3 \beta$ phosphorylation in the PBMCs from the T2D patients were significantly lower than those in the HC group. Notably, the relative expression levels of Nurrl were correlated negatively with the levels of plasma IL- 6 and TNF- $\alpha$, as well as the degree of insulin resistance, in the T2D patients. To the best of our knowledge, this is the first report to demonstrate that significantly reduced expression levels of Nurrl in PBMCs were negatively correlated with the levels of pro-inflammatory cytokines in the plasma and with the degrees of insulin resistance in T2D patients. Previous studies have reported lower expression levels of Nurrl in patients with neuroimmunological and inflammatory disorders, including Parkinson's disease and metabolic syndrome $(9,10,12,33,34)$. The findings of the present study extended those of previous observations and suggested that lower expression levels of Nurr1 may be present in patients with other types of inflammatory diseases. Given that insulin resistance is crucial for the development of T2D and other metabolic syndrome-associated chronic inflammatory diseases, the significant inverse correlation identified between the expression of Nurr1 and insulin resistance suggested that the expression of Nurr1 in PBMCs may be a biomarker for clinically evaluating the degrees of insulin resistance in T2D and other insulin-resistance-associated metabolic disorders (31).

It is well established that high levels of glucose and saturated fat acid can stimulate inflammatory cytokine production in human immunocompetent cells (28-30). In the present study, high levels of glucose and palmitic acid inhibited the expression of Nurrl in the PBMCs from healthy subjects in vitro, in a dose- and time-dependent manner. Previous studies have suggested that Nurrl, together with its co-factor of phosphorylated GSK- $3 \beta$ and CoREST can interact with the NF-kB transcription factor in the promoters of inflammatory cytokines to inhibit their transcription $(19,20)$. NR4A3 and NR4A1, members of the same family as Nurr1, have been observed to regulate the membrane translocation of glucose transporter of GLUT4 and insulin receptor phosphorylation in muscular cells, and enhances insulin sensitivity (11). The present study demonstrated significantly lower expression levels of Nurrl and GSK-3 $\beta$ phosphorylation in the PBMCs from T2D patients. It is possible that metabolic disorder-associated high levels of glucose and high levels of saturated fatty acids inhibit the expression of Nurrl and GSK- $3 \beta$ phosphorylation, to mitigate their inhibition on NF- $\kappa \mathrm{B}$ activation, leading to increased levels of pro-inflammatory cytokine production, particularly for TNF- $\alpha$. High levels of pro-inflammatory cytokines, together with lower expression levels of NR4A members inhibit glucose transporter expression and insulin receptor phosphorylation, leading to insulin resistance. Long term insulin resistance results in hyperglycemia and hyperlipidemia, which further downregulate the expression of Nurr1, creating positive feedback regulation and driving the pathogenesis of T2D. Further investigation of the regulatory effect of Nurrl on inflammation, insulin resistance and T2D development is suggested.

The present study had limitations of a small sample size and the lack of longitudinal observations and investigation of the molecular mechanisms underlying the regulation of Nurrl on inflammation and insulin resistance. Therefore, further longitudinal studies in a larger population are warranted.

In conclusion, the present study demonstrated significantly higher levels of pro-inflammatory cytokines, but lower expression levels of Nurrl and GSK-3 $\beta$ phosphorylation in PBMCs from T2D patients. Furthermore, the expression levels of Nurrl in the PBMCs were negatively correlated with 
the degree of insulin resistance in the T2D patients. High levels of glucose and palmitic acid inhibited the expression of Nurr1 in the PBMCs from healthy subjects in vitro, in a dose- and time-dependent manner. These data suggested that the expression levels of Nurrl may serve as a biomarker for evaluating the severity of insulin resistance. These findings may provide novel insight in the pathogenesis of chronic inflammation-associated insulin resistance and T2D.

\section{Acknowledgements}

This study was supported by grants from the National Natural Science Foundation of China (grant. nos. 81170769 and 81370872).

\section{References}

1. Tuomi T, Santoro N, Caprio S, Cai M, Weng J and Groop L: The many faces of diabetes: a disease with increasing heterogeneity. Lancet 383: 1084-1094, 2014.

2. Ma RC and Chan JC: Type 2 diabetes in east Asians: similarities and differences with populations in Europe and the United States. Ann N Y Acad Sci 1281: 64-91, 2013.

3. Cildir G, Akincilar SC and Tergaonkar V: Chronic adipose tissue inflammation: all immune cells on the stage. Trends Mol Med 19: 487-500, 2013.

4. Tilg $\mathrm{H}$ and Moschen AR: Inflammatory mechanisms in the regulation of insulin resistance. Mol Med 14: 222-231, 2008.

5. Osborn $\mathrm{O}$ and Olefsky JM: The cellular and signaling networks linking the immune system and metabolism in disease. Nat Med 18: 363-374, 2012.

6. Feuerer M, Herrero L, Cipolletta D, et al: Lean, but not obese, fat is enriched for a unique population of regulatory $\mathrm{T}$ cells that affect metabolic parameters. Nat Med 15: 930-939, 2009.

7. DeFuria J, Belkina AC, Jagannathan-Bogdan M, et al: B cells promote inflammation in obesity and type 2 diabetes through regulation of T-cell function and an inflammatory cytokine profile. Proc Natl Acad Sci USA 110: 5133-5138, 2013.

8. Navarro-Gonzalez J, Mora-Fernandez C, Gomez-Chinchon M, Muros M, Herrera H and Garcia J: Serum and gene expression profile of tumor necrosis factor-alpha and interleukin-6 in hypertensive diabetic patients: effect of amlodipine administration. Int J Immunopathol Pharmacol 23: 51-59, 2010.

9. Hamers AA, Hanna RN, Nowyhed H, Hedrick CC and de Vries CJ: NR4A nuclear receptors in immunity and atherosclerosis. Curr Opin Lipidol 24: 381-385, 2013.

10. Pearen MA and Muscat GE: Minireview: Nuclear hormone receptor 4A signaling: implications for metabolic disease. Mol Endocrinol 24: 1891-1903, 2010.

11. Fu Y, Luo L, Luo N, Zhu X and Garvey WT: NR4A orphan nuclear receptors modulate insulin action and the glucose transport system: potential role in insulin resistance. J Biol Chem 282: 31525-31533, 2007.

12. Bonta PI, Pols TW, van Tiel CM, et al: Nuclear receptor Nurrl is expressed in and is associated with human restenosis and inhibits vascular lesion formation in mice involving inhibition of smooth muscle cell proliferation and inflammation. Circulation 121: 2023-2032, 2010.

13. McEvoy AN, Bresnihan B, Fitzgerald O and Murphy EP: Corticotropin-releasing hormone signaling in synovial tissue vascular endothelium is mediated through the cAMP/CREB pathway. Ann N Y Acad Sci 966: 119-130, 2002.

14. Zhang Z, Li X, Xie WJ, et al: Anti-parkinsonian effects of Nurr1 activator in ubiquitin-proteasome system impairment induced animal model of Parkinson's disease. CNS Neurol Disord Drug Targets 11: 768-773, 2012.
15. Block Ml, Zecca L and Hong JS: Microglia-mediated neurotoxicity: uncovering the molecular mechanisms. Nat Rev Neurosci 8: 57-69, 2007.

16. Bonta PI, van Tiel CM, Vos M, et al: Nuclear receptors Nur77, Nurr1 and NOR-1 expressed in atherosclerotic lesion macrophages reduce lipid loading and inflammatory responses. Arterioscler Thromb Vasc Biol 26: 2288-2294, 2006.

17. Wang T, Jiang Q, Chan C, et al: Inhibition of activation-induced death of dendritic cells and enhancement of vaccine efficacy via blockade of MINOR. Blood 113: 2906-2913, 2009.

18. Buss H, Dörrie A, Schmitz Ml, et al: Phosphorylation of serine 468 by GSK-3beta negatively regulates basal p65 NF-kappaB activity. J Biol Chem 279: 49571-49574, 2004.

19. Saijo K, Winner B, Carson CT, et al: A Nurr1/CoREST pathway in microglia and astrocytes protects dopaminergic neurons from inflammation-induced death. Cell 137: 47-59, 2009.

20. Wan F and Lenardo MJ: The nuclear signaling of NF-kappaB: current knowledge, new insights and future perspectives. Cell Res 20: 24-33, 2010

21. McMorrow JP and Murphy EP: Inflammation: a role for NR4A orphan nuclear receptors? Biochem Soc Trans 39: 688-693, 2011.

22. O'Kane M, Murphy EP and Kirby B: The role of corticotropin-releasing hormone in immune-mediated cutaneous inflammatory disease. Exp Dermatol 15: 143-153, 2006.

23. Pei L, Castrillo A, Chen M, Hoffmann A and Tontonoz P: Induction of NR4A orphan nuclear receptor expression in macrophages in response to inflammatory stimuli. J Biol Chem 280: 29256-29262, 2005.

24. Safe S, Jin UH, Hedrick E, Reeder A and Lee SO: Minireview: role of orphan nuclear receptors in cancer and potential as drug targets. Mol Endocrinol 28: 157-172, 2014.

25. Expert Committee on the Diagnosis and Classification of Diabetes Mellitus: Report of the expert committee on the diagnosis and classification of diabetes mellitus. Diabetes Care 26 (Suppl 1): S5-S20, 2003.

26. Turner RC, Holman RR, Matthews D, Hockaday TD and Peto J: Insulin deficiency and insulin resistance interaction in diabetes: Estimation of their relative contribution by feedback analysis from basal plasma insulin and glucose concentrations. Metabolism 28: 1086-1096, 1979.

27. Livak KJ and Schmittgen TD: Analysis of the relative gene expression data using real-time quantitative PCR and the 2(-Delta Delta C(T)) Method. Methods 25: 402-408, 2001.

28. Hâncu N, Netea MG and Baciu I: High glucose concentrations increase the tumor necrosis factor-alpha production capacity by human peripheral blood mononuclear cells. Rom J Physiol 35: 325-330, 1998

29. Fogeda M, Gallart L, Gutierrez C, et al: High expression of tumor necrosis factor alpha receptors in peripheral blood mononuclear cells of obese type 2 diabetic women. Eur Cytokine Netw 15: 60-66, 2004.

30. Mylona EE, Mouktaroudi M, Crisan TO, et al: Enhanced interleukin-1beta production of PBMCs from patients with gout after stimulation with Toll-like receptor-2 ligands and urate crystals. Arthritis Res Ther 14: R158, 2012.

31. D'Amore S, Vacca M, Graziano G, et al: Nuclear receptors expression chart in peripheral blood mononuclear cells identifies patients with metabolic syndrome. Biochim Biophys Acta 1832: 2289-2301, 2013.

32. Giulietti A, Stoffels K, Decallonne B, Overbergh L and Mathieu C: Monocytic expression behavior of cytokines in diabetic patients upon inflammatory stimulation. Ann N Y Acad Sci 1037: 74-78, 2004.

33. Davies MR, Harding CJ, Raines S, et al: Nurrl dependent regulation of pro-inflammatory mediators in immortalised synovial fibroblasts. J Inflamm (Lond) 2: 15, 2005.

34. Lee JK, Tran T and Tansey MG: Neuroinflammation in Parkinson's disease. J Neuroimmune Pharmacol 4: 419-429, 2009. 